\title{
Scientific Oral Presentation Abstracts
}

\section{• 10.5812/IRANJRADIOL.99140}

\section{Deep Learning Approach for Left Atrium Segmentation}

Shakiba Moradi ${ }^{1}$; Mostafa Ghelich Oghli², *;Azin Alizadehasl³; Ali Shabanzadeh ${ }^{2}$

${ }^{1}$ Sharif University of Technology, Tehran, Iran

${ }^{2}$ Intelligent Imaging Technology Research Center, Med Fanavarn Plus Co., Karaj, Iran

${ }^{3}$ Rajaie Cardiovascular Medical and Research Center, Iran University of Medical Science, Tehran, Iran

${ }^{*}$ Corresponding author: Intelligent Imaging Technology Research Center, Med Fanavarn Plus Co., Karaj, Iran.Email:m.g31_mesu@yahoo.com

\section{Abstract}

Background:Cardiovascular disease is one of the most common causes of death in the world. Cardiovascular risk is often assessed by analyzing parameters extracted from image data taken from different parts of the heart. Transthoracic echocardiography is a non-invasive imaging modality that is usually the first step of diagnosis procedure. Extracting more information from epical and parasternal views can lead the doctor to early diagnosis. The examination analysis, although has a valuable role, relies on the operator experience. To have a more standard analysis, it is important to reduce the variability of the examination analysis. Automatic image analysis, which is very helpful in this context, can be implemented in image segmentation, parameter measurement, and even diagnosis levels. Left atrial measurements (mostly the atrial volume) are strong predictors of cardiac events. Elevated atrial pressure or increased flow can lead to atrial enlargement. There is evidence that patients with high left atrial volume are at risk of ischemic stroke, heart failure, and atrial fibrillation.

Objectives: The purpose of this paper was to conduct automatic segmentation of left atrium in four-chamber view images to make measurements reliable and independent from expert experience. Generally, most methods of automatic segmentation are based on image processing algorithms that are sometimes too complicated and less accurate. Deep learning models have been widely used in different computer vision areas. These models are mathematically simple and are proven to have better accuracy in computer vision problems. Accordingly, we used one of the famous neural network structures named Unet for left atrium segmentation in $2 \mathrm{D}$ echocardiography. Comparing the trained network's results with the ground truth segmentations made by experts, we showed that neural networks were strong choices for automation.

Methods: The dataset used in this research was the public echocardiography image dataset published in CAMUS (Cardiac Acquisitions for Multi-structure Ultrasound Segmentation) Challenge. The data included four-chamber view end-systole and end-diastole frames from 450 patients. Unet is a kind of pyramidal network with encoding and decoding paths. The encoder or contraction path was used to capture the context and features in the image. The second path was the symmetric expanding or decoder path that was used to enable precise localization. The whole task of the network was to classify each pixel in the image to the background or left atrium classes. The loss function in the learning procedure was defined as the Dice coefficient, which depicted the ratio of the number of pixels that were correctly classified. For comparing the method with experts, five-fold cross-validation was used. Each time, $10 \%$ of the dataset was used as a test set and $90 \%$ as a train set. In the training step, the network learned the train set annotations and in the testing procedure, the performance of the trained network was evaluated based on its predictions on the test set. Dice coefficient, Hausdorff distance, and mean absolute distance (MAD) were used to evaluate the accuracy of the method in echocardiography images.

Results: The calculated metrics were dice coefficient of 91.17\%, Hausdorff distance of 3.31, and MAD of 0.71 for the train set and dice coefficient of $88.14 \%$, Hausdorff distance of 4.75, and MAD of 1.67 for the test set. Conclusion: We showed that using Unet architecture in four-chamber view echocardiography images for bordering left atrium can be a powerful alternative to remove the variability between different experts. Evaluation metrics analysis proved that we can rely on the method predictions and have a more accurate shape of the left atrium for further analysis and measurements such as volume and other features measurement.

\section{-10.5812/IRANJRADIOL.99142}

\section{Skin Lesion Diagnosis Using Ensemble Deep Learning Models}

Mehdi Yousefzadeh ${ }^{1,2}$; Parsa Esfahanian ${ }^{1, *}$; Saeid Rahmani ${ }^{1}$; Hossein Motahari' ${ }^{2}$ : Dara Rahmati ${ }^{1,3}$; Saeid Gorgin ${ }^{1,4}$

\footnotetext{
${ }^{1}$ School of Computer Science, Institute for Research in Fundamental Sciences, Tehran, Iran

${ }^{2}$ Faculty of Physics, Shahid Beheshti University, Tehran, Iran
} 


\section{Scientific Oral Presentation Abstracts}

\footnotetext{
${ }^{3}$ Faculty of Computer Science and Engineering, Shahid Beheshti University, Tehran, Iran

${ }^{4}$ Iranian Research Organization for Science and Technology, Tehran, Iran

${ }^{*}$ Corresponding Author: School of Computer Science, Institute for Research in Fundamental Sciences, Tehran, Iran. Email: parsa.esfahanian.7596@gmail.com
}

\begin{abstract}
Background: Skin cancer is a serious public health concern (1). With over 5 million newly diagnosed cases every year, it is the most common form of cancer worldwide (2,3). Among the different categories of skin cancer, melanoma is the deadliest and most dangerous form. It is estimated to be responsible for 7230 cases of death in 2019 globally (4). Although the melanoma mortality rate is significant, its survival rate exceeds $95 \%$ if detected early $(5,6)$.

Objective: We propose a deep learning framework that could perform skin lesion diagnosis with precision and accuracy. Such a framework could work by classifying dermoscopic images based on skin lesion categories. These categories include melanoma, melanocytic nevus, basal cell carcinoma, actinic keratosis, benign keratosis, dermatofibroma, and vascular lesion.
\end{abstract}

Method: We used the convolutional neural network models to classify dermoscopic images. The selected models had high Top-1 accuracy on the ImageNet dataset. These models included InceptionResNetV2, Xception, and EfficientNetB3. All of these models were initialized with their pre-trained weights on the ImageNet dataset. They all used categorical cross-entropy for loss function and Adam optimizer with standard parameters. Two different ensemble methods were employed in our study. The first one, called Softmax-only, used Xception and EfficientNetB3 with Softmax activation for both models' prediction layer. In this method, during the training process, each model created a checkpoint of itself and recorded its balance accuracy at each epoch. After training completion, checkpoints corresponding to some of the highest balance accuracies for each model would be selected. The average balance accuracy of the selected model checkpoints was reported as the method's performance. The second method was called Sigmoid-only that used InceptionResNetV2, Xception, and EfficientNetB3. This method performed pretty much the same as the first, except that all the models had Sigmoid activation for their prediction layers and the evaluation criterion was the F1-score. The used dataset was the 2018 ISIC archive, which included 25331 dermoscopic images for the training set and 1516 images for the test (evaluation) set (1). For the Softmax-only method, the training set was split $80 \%-20 \%$ for crossvalidation and for the Sigmoid-only method, it was split $85 \%$ - $15 \%$ for fixed-validation. The training set was also augmented using random crop, random rotation, and random flipping.

Results: The Softmax-only method had a balanced accuracy of 0.901 ( \pm 0.12) and the Sigmoid-only method had an average F1-score of 0.932 on the seven classes. Finally, our framework managed to achieve a balanced accuracy of 0.866 on the test set. Furthermore, our framework participated in Task 3 of the "ISIC 2018: Skin Lesion Analysis Towards Melanoma Detection" challenge, which brought us the first rank on the challenge's live leaderboards (up until 18 September 2019; link: https://challenge2018. isic-archive.com/live-leaderboards/).

Conclusion: We proposed a deep learning framework to classify the ISIC archive 2018 dataset of dermoscopic images based on skin lesion categories. Our methods had the validation results of 0.901 ( \pm 0.12 ) for balance accuracy and 0.932 for average F1score, as well as a balanced accuracy of 0.866 on the test set. This framework also managed to achieve the first rank of the "ISIC 2018 Task 3" challenge.

$\begin{aligned} & \text { Table 1. Description and Average Balance Accuracy of Models } \\
& \text { in the Softmax-Only Ensemble Method }\end{aligned}$
\begin{tabular}{lcc}
\hline Model & Input Size & Balance Accuracy \\
\hline Xception & $299 \times 299$ & $0.864 \pm 0.011$ \\
\hline EfficientNetB3 & $300 \times 300$ & $0.882 \pm 0.014$ \\
\hline Softmax-Only Ensemble & - & $0.901 \pm 0.012$ \\
\hline
\end{tabular}

Table 2. Classes and Best F1-Score of the Sigmoid-Only Ensemble Method

\begin{tabular}{lc}
\hline Class & F1-score \\
\hline Melanoma & 0.882 \\
\hline Melanocytic nevus & 0.958 \\
\hline Basal cell carcinoma & 0.960 \\
\hline Actinic keratosis & 0.887 \\
\hline Benign Keratosis & 0.881 \\
\hline Dermatobroma & 0.989 \\
\hline Vascular lesion & 0.969 \\
\hline
\end{tabular}

\section{References}

1. ISIC 2018 Challenge. ISIC Challenge 2018 Leaderboards. In: challenge2018.isic-archive.com (2018). URL: https://challenge2018.isic-archive.com/leaderboards/.

2. Guy GP, Machlin S, Ekwueme DU, Yabro_KR. Prevalence and costs of skin cancer treatment in the US, 20022006 and 20072011. Am J Prev Med. 2015; 48:1837.

3. https://cancerstatisticscenter.cancer.org/\#!/cancer-site/Melanoma\%20of\%20the\%20skin. 


\section{SCientific Oral Presentation Abstracts}

4. https://www.cancer.org/research/cancer-facts-statistics/allcancer-facts-figures/ cancer-facts-figures-2018.html.

5. https://dermoscopedia.org/Melanoma.

6. https://dermoscopedia.org/Benign_Melanocytic_lesions.

\section{-10.5812/IRANJRADIOL. 99143}

\section{Automatic Assessment of Ki-67 Proliferation Index in Lymphoma}

Ramin Nateghi'1, *; Mansoor Fatehi' ${ }^{2}$; Nasrin Shayanfar ${ }^{3}$; Fattane Pourakpour ${ }^{2}$; Wolfgang Pokieser ${ }^{4}$; Christophe Kornauth ${ }^{5}$

${ }^{1}$ Department of Electrical and Electronic Engineering, Shiraz University of Technology, Shiraz, Iran

${ }^{2}$ National Brain Mapping Lab, Tehran, Iran

${ }^{3}$ Department of Pathology, Iran University of Medical Sciences, Tehran, Iran

${ }^{4}$ Klinisches Institut für Pathologie, Medizinische Universität Wien, Vienna, Austria

${ }^{5}$ Labor für Klinische Zytologie, Institut für Pathologie des Wihelminenspials, Vienna, Austria

${ }^{*}$ Corresponding author: Department of Electrical and Electronic Engineering, Shiraz University of Technology, Shiraz, Iran. Email: r.nateghi.s@gmail.com

\begin{abstract}
Background:Haematopathological Ki-67 is used principally to measure the proliferation rate in the assessment and grading of malignancies. Ki-67 is based on a powerful staining method for distinguishing benign from malignant proliferation. The index uses a nuclear protein expression and it has been widely used to evaluate the proliferative activity of lymphoma. The clinical value of Ki-67 includes defining prognosis (among lymphomas), predicting drug response, and setting eligibility criteria for clinical trials. The Ki-67 score or index should be expressed as the percentage of positively stained cells among the total number of invasive cells in the area scored. With the Ki-67 marker, the proliferation fraction of low-grade follicular lymphomas (FLs) is usually less than 20\% (as shown here) and that of
\end{abstract}

high-grade FLs is greater than 30\% [1]. Manual Ki-67 proliferation assessment is a very time-consuming and operator-dependent task at the same time. Therefore, several studies have examined the use of image analysis software to measure faster the nuclear staining index of Ki-67 in lymphomas. A few studies have focused on the measurement of proliferation index in FLs and found that automated Ki-67 counts were similar to manual counts [2-3]. A major source of difference between automatic and manual Ki-67 scores is the scoring method that depends on the strategy of counting or the estimation and choice of the area to count.

Methods: In this research, an automatic unsupervised learning-based system was proposed for accurate and fast Ki-67 scoring in lymphoma. The proposed methods were designed to use image processing tools and detect robustly the positive and negative cells for Ki-67 antibody. The goal of the proposed method was to assess the proliferation index (percentage of Ki-67 positive lymphoma cells) to provide better treatment options for lymphoma patients. The proposed system consisted of the following sections: pre-processing, feature extraction, segmentation, and post-processing (Figure 1). To highlight specific histological structures of Ki-67 stained images such as positives cells (brown color ones), we performed pre-processing such as color transform from RGB space to brown-ratio space. For smoothing and filling the region of each cell on the image, the morphological filling was used. After the pre-processing section, color features, such as the mean of brown-ratio color space and blue channel of RGB image in a $3 \times 3$ block, were extracted from the image. In the next section of the proposed system, using the extracted color features, the image was segmented into three clusters by k-means clustering. After image segmentation, each of the positive and negative cells was post-processed. In the post-processing section, to split the merged cell, the morphological opening was used and finally, false segmented regions with small areas were removed.

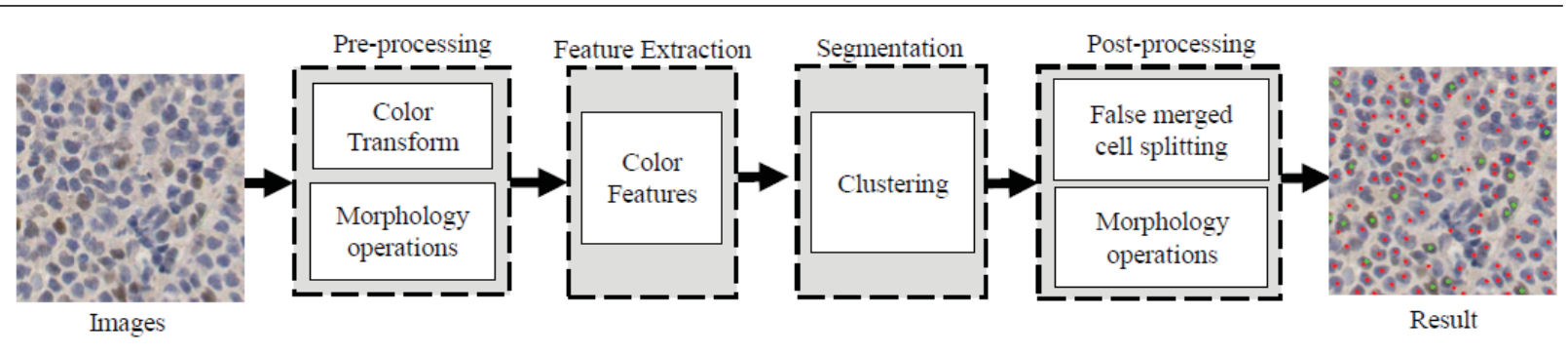

Figure 1. The proposed automatic Ki-67 assessment system 